\title{
The nuclear reaction model code MEDICUS
}

\author{
Agim I. Ibishi ${ }^{\text {a }}$ \\ Cyclotron Department, Vrije Universiteit Brussel, Laarbeeklaan 103, 1090 Brussels, Belgium
}

\begin{abstract}
The new computer code MEDICUS has been used to calculate cross sections of nuclear reactions. The code, implemented in MATLAB 6.5, Mathematica 5, and FORTRAN 95 programming languages, can be run in graphical and command line mode. Graphical User Interface (GUI) has been built that allows the user to perform calculations and to plot results just by mouse clicking. The MS Windows XP and Red Hat Linux platforms are supported. MEDICUS is a modern nuclear reaction code that can compute charged particle-, photon-, and neutroninduced reactions in the energy range from thresholds to about $200 \mathrm{MeV}$. The calculation of the cross sections of nuclear reactions are done in the framework of the Exact Many-Body Nuclear Cluster Model (EMBNCM), Direct Nuclear Reactions, Pre-equilibrium Reactions, Optical Model, DWBA, and Exciton Model with Cluster Emission. The code can be used also for the calculation of nuclear cluster structure of nuclei. We have calculated nuclear cluster models for some nuclei such as ${ }^{177} \mathrm{Lu},{ }^{90} \mathrm{Y}$, and ${ }^{27} \mathrm{Al}$. It has been found that nucleus ${ }^{27} \mathrm{Al}$ can be represented through the two different nuclear cluster models: ${ }^{25} \mathrm{Mg}+\mathrm{d}$ and ${ }^{24} \mathrm{Na}+{ }^{3} \mathrm{He}$. Cross sections in function of energy for the reaction ${ }^{27} \mathrm{Al}\left({ }^{3} \mathrm{He}, \mathrm{x}\right){ }^{22} \mathrm{Na}$, established as a production method of ${ }^{22} \mathrm{Na}$, are calculated by the code MEDICUS. Theoretical calculations of cross sections are in good agreement with experimental results. Reaction mechanisms are taken into account.
\end{abstract}

\section{Introduction}

The MEDICUS code was initially implemented as a code for the modelling of nuclear reactions which are often used for production of biomedical radioisotopes [1,14,15]. Later on, the usage of MEDICUS was extended to nuclear cluster structure calculations [2], imaging, visualisation and image processing. The current nuclear reaction theories and models are implemented in the code. The code can be run in graphical and command line mode. The MS Windows XP and Red Hat Linux platforms are supported. Graphical User Interface (GUI) has been built, that allows user to perform calculations and to plot results just by mouse clicking. The MEDICUS code contains and uses scripts and functions written in MATLAB 6.5, Mathematica 5, and FORTRAN 95 programming languages. MEDICUS also includes newer object-oriented programming constructs. Special attention has been paid for the calculation of cross sections of nuclear reactions which are relevant to the production of nuclear isomers. Nuclear isomers of an element are nuclides with the same atomic number and same mass number, but with a different nuclear cluster structure [3], e.g., with a different energy state [3-5, 11, 12].

The internal nuclear cluster wave functions of different nuclear cluster models (nuclear cluster isomers) of the same isotope are not equivalent if we take into account manybody forces, such as 3-body forces and 4-body forces [3,4, $6,7]$. Nuclear isomers of an element show the same chemical and biological behavior, but a different nuclear and radiation activity. Since the standard nuclear reaction theories are well known, in this article we will pay more attention to the newest theory: the Exact Many-Body Nuclear Cluster Model (EMBNCM). Furthermore, the recent developments intend to

\footnotetext{
${ }^{a}$ Present address, Faculty of Science, Free University of Brussels, Nieuwelaan 149/b 529, 1040 Bruxelles, Belgium

e-mail: aibishi@vub.ac.be
}

extend application of the MEDICUS code to its usage for the general purposes in nuclear physics, biomedical engineering, and in nuclear medicine. In section 2 we begin by explaining the methods used for implementation of the MEDICUS code, both physics and the programming. Then in section 3 we present some important results, e.g., cross section calculations, and nuclear cluster structure calculations.

\section{Methods}

\subsection{Physics and theoretical models}

MEDICUS is a modern nuclear reaction code that can compute charged particle-, photon-, and neutron- induced reactions in the energy range from thresholds to about $200 \mathrm{MeV}$. The calculations of cross sections of nuclear reactions are done in the framework of the Exact Many-Body Nuclear Cluster Model, Direct Nuclear Reactions, Pre-equilibrium Reactions, Optical Model, DWBA, and Exciton Model with Cluster Emission. Several other improvements are incorporated in the calculation approaches.

Using the EMBNCM model, Pre-equilibrium Theory, Direct Reactions, and the Exciton Model, we can write the cross sections formula for the nuclear reaction $(a, b)$ in the form

$$
\frac{d \sigma_{a, b}(E)}{d E_{a}}=\sigma_{a}\left(E_{a}\right) K_{C-G}^{2} \sum_{f} \sum_{f \pi} T(f, f \pi, I) R_{b} .
$$

where

$$
K_{C-G}=\sum_{I}\left[C_{a}\left(I, I_{a}\right)\right]
$$

are the isospin coupling Clebsch-Gordan coefficients, $\sigma_{a}$ is the cross section for forming the composite nucleus, $I$ is the 
isospin, $R_{b}$ is the emission rate at exit channel, $E_{a}$ is evaluated at the incident channel energy, $T$ is the time of the reaction process, and $f, f_{\pi}$ are the strength functions like in refs. $[3,4,8-10]$. The form of the wave functions is derived like in refs. $[3,4]$.

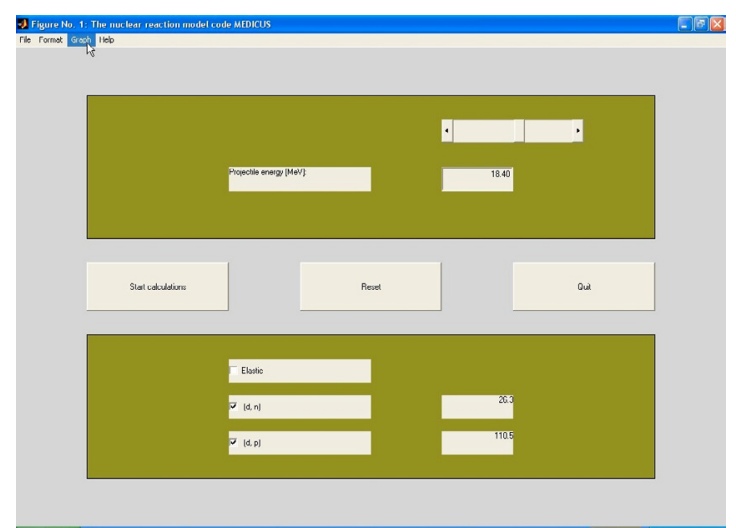

Fig. 1. The MEDICUS toolbox for MATLAB, called code NOAH.

\subsection{Programming details and implementation of the code}

The MEDICUS code has been implemented in Mathematica 5, FORTRAN 95, and MATLAB 6.5 programming languages. The MEDICUS toolbox for MATLAB 6.5 programming language, called code NOAH, and the interface has been built. They integrate modelling of nuclear reactions, imaging, visualization, image processing, and computing into the MATLAB 6.5 environment. The code NOAH is implemented as a MEDICUS toolbox for MATLAB 6.5, and it is illustrated in figure 1. The NOAH code can be run in command line mode, and in Interface mode. The code MEDICUS contains traditional programming constructs, as well as matrix-oriented functions and operators. The traditional procedural programming as well as object-oriented programming has been used during the implementation of the MEDICUS code. The main code MEDICUS integrates several other codes and scripts that can be run independently, as well as within the main code MEDICUS. The code NOAH can perform cross section calculations for a large class of nuclear reactions.

\section{Results}

\subsection{Calculation of nuclear cluster structure of nuclei}

The code MEDICUS can be used through the TRINITY code, for calculation of the nuclear cluster structure of nuclei. We have calculated nuclear cluster models for some nuclei such as: ${ }^{27} \mathrm{Al},{ }^{90} \mathrm{Y}$, and ${ }^{177} \mathrm{Lu}$. It has been found that the nucleus ${ }^{27} \mathrm{Al}$ can be represented through the two different nuclear cluster models: ${ }^{25} \mathrm{Mg}+\mathrm{d}$, and ${ }^{24} \mathrm{Na}+{ }^{3} \mathrm{He}$. We follow here the analysis first described in refs. [3,4]. We write the nuclear wave function for the A-nucleon system in the form $\Psi=$ $\Psi\left(\tilde{r}_{1}, \ldots, \tilde{r}_{A} ; t\right)$, where $\tilde{r}_{i}$ denotes the space, spin, and isobaricspin coordinates of the $i^{\text {th }}$ nucleon. The EMBNCM accounts quantitatively for many features of both light and heavy nuclei. Solving the Schrödinger equation for the relative motion of the two, three, or four sub-nuclei, the exterior cluster(s) and the core cluster, in potential well which has a Gaussian form gives rise to a rotational band of states. Using the Exact Many-Body Nuclear Cluster Model, the ground state of ${ }^{27} \mathrm{Al}$ is described by the following two different nuclear cluster wave functions:

$$
\Psi_{g s}=N_{0} \Phi_{0}\left({ }^{25} M g\right) \Phi_{0}(d) K_{00}(\tilde{R}) .
$$

and

$$
\Psi_{g s}^{\prime}=N_{0} \Phi_{0}\left({ }^{24} \mathrm{Na}\right) \Phi_{0}\left({ }^{3} \mathrm{He}\right) K_{00}^{\prime}(\tilde{R}) .
$$

The nuclear cluster wave functions $K_{00}(\tilde{R})$ and $K_{00}^{\prime}(\tilde{R})$ describe the relative motion of the two clusters. We assume that the exterior cluster is in its ground state. The exterior cluster nucleons must occupy levels above the Fermi surface of the core cluster in order to satisfy the Pauli exclusion principle. The $\Phi_{0}\left({ }^{25} \mathrm{Mg}\right), \Phi_{0}(d), \Phi_{0}\left({ }^{24} \mathrm{Na}\right)$, and $\Phi_{0}\left({ }^{3} \mathrm{He}\right)$ are the internal nuclear cluster wave functions, and $\mathrm{N}_{0}$ is a constant. In the EMBNCM model, states in a nucleus are assumed to correspond to two, three, or four sub-nuclei orbiting each other in a deep potential well. The shape of the potential well has a Gaussian form which is the shape generated by means of a double-folding calculation. The wave functions $\Psi_{g s}$ and $\Psi_{g s}^{\prime}$ are not equivalent if we take into account threebody forces (3BF), and four-body forces (4BF). Indeed in the wave function $\Psi_{g s}$ appear two-body forces (2BF) and threebody forces $(3 \mathrm{BF})$, and in the wave function $\Psi_{g s}^{\prime}$ appear $2 \mathrm{BF}$, $3 \mathrm{BF}$, and additional $4 \mathrm{BF}$. The projection of the wave function $\Phi_{0}\left({ }^{25} \mathrm{Mg}\right) \Phi_{0}(d)$ on $\Phi_{0}\left({ }^{24} \mathrm{Na}\right) \Phi_{0}\left({ }^{3} \mathrm{He}\right)$ would be zero. Furthermore, functions $\Psi_{g s}$ and $\Psi_{g s}^{\prime}$ are not orthogonal. Hartree-Fock variational calculations for nuclei such as ${ }^{27} \mathrm{Al}$ indicate that there are indeed states which look like two, three, or four clusters, providing a justification for this assumption. The similar proof for the non-equivalence of nuclear cluster wave functions of different cluster models can be shown for the ${ }^{90} \mathrm{Y}$, and ${ }^{177} \mathrm{Lu}$ nuclei. In the internal nuclear cluster wave functions, we have to replace the coordinates $\tilde{r}_{i}$ of the $i^{\text {th }}$ nucleon, with Jacobi coordinates. For this purposes a new system of Jacobi coordinates has been developed. The allowed values of the principal quantum number $P$ and the angular momentum $L$ for rotational states are expressed by formula (5) by having a constant value of

$$
2 P+L=\sum_{j=1}^{n_{c}}\left(2 P_{j}+l_{j}\right) .
$$

where $p_{j}$ and $l_{j}$ are single particle quantum numbers of the exterior cluster, and $n_{c}$ is the number of exterior cluster nucleons. For $\Psi_{g s}$, the $n_{c}=2$ and for $\Psi_{g s}^{\prime}$, the $n_{c}=3$. The code MEDICUS include the TRINITY code designed for calculation of nuclear cluster structure of nuclei. The TRINITY code is written in Mathematica 5, MATLAB 6.5, and FORTRAN 95 programming languages.

\subsection{Cross section calculations}

In modelling of specific nuclear reactions used for production of biomedical radioisotopes, new results were achieved. 


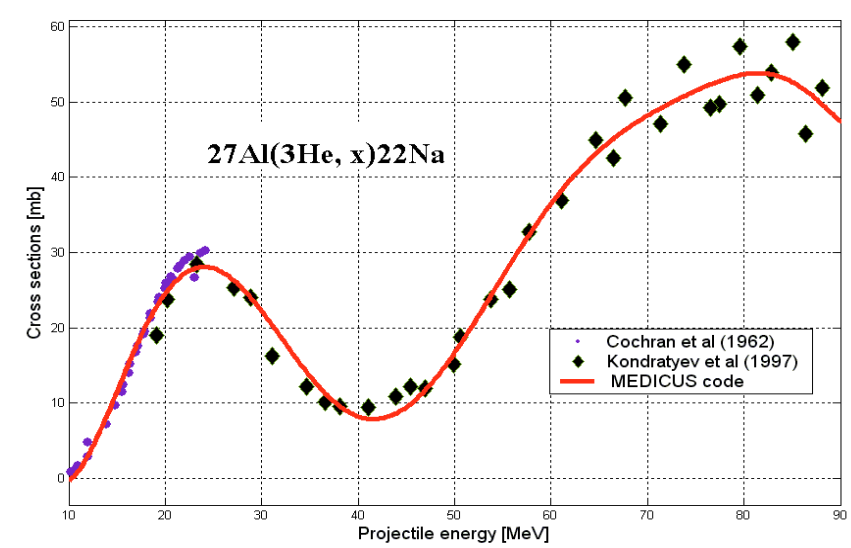

Fig. 2. Theoretical calculations of cross sections for the reaction ${ }^{27} \mathrm{Al}\left({ }^{3} \mathrm{He}, \mathrm{x}\right){ }^{22} \mathrm{Na}$ from MEDICUS code (line), and experimental data by Cochran et al. (1962), and Kondratyev et al. (1997) $\left({ }^{\circ}\right.$, symbols).

Table 1. Calculated cluster models for ${ }^{27} \mathrm{Al},{ }^{90} \mathrm{Y}$, and ${ }^{177} \mathrm{Lu}$ nuclei.

\begin{tabular}{|c|c|c|c|}
\hline Isotope & ${ }^{27} \mathrm{Al}$ & ${ }^{90} \mathrm{Y}$ & ${ }^{177} \mathrm{Lu}$ \\
\hline Cluster model & ${ }^{25} \mathrm{Mg}+\mathrm{d}$ & ${ }^{87} \mathrm{Rb}+{ }^{3} \mathrm{He}$ & ${ }^{176} \mathrm{Yb}+{ }^{1} \mathrm{H}$ \\
\hline & ${ }^{24} \mathrm{Na}+{ }^{3} \mathrm{He}$ & & \\
\hline
\end{tabular}

Cross sections in function of energy for the reaction ${ }^{27} \mathrm{Al}\left({ }^{3} \mathrm{He}, \mathrm{x}\right){ }^{22} \mathrm{Na}$, established as a production method of ${ }^{22} \mathrm{Na}$, are calculated by the MEDICUS code and are shown in figure 2. For this calculation have been used the EMBNCM model with Coulomb interaction for the charge exchange reactions, the Pre-equilibrium model, DWBA, the theory of Direct Nuclear Reactions, and the Fermi Gas (FG) model. The formula for cross section calculations is given by equation (1). For the description of this reaction many-body formalism developed on EMBNCM were found to give accurate results [3]. In table 1 are presented calculated cluster models for the ${ }^{27} \mathrm{Al}$ nucleus. During the reactions ${ }^{27} \mathrm{Al}\left({ }^{3} \mathrm{He}, \mathrm{x}\right)^{22} \mathrm{Na}$, and ${ }^{27} \mathrm{Al}\left({ }^{3} \mathrm{He}, \mathrm{x}\right){ }^{24} \mathrm{Na}$, we have in the exit channel two different products: ${ }^{22} \mathrm{Na}$ and ${ }^{24} \mathrm{Na}$. We will treat this class of reactions in the framework of the EMBNCM model. According to the EMBNCM model there are two different nuclear cluster models for the nucleus ${ }^{27} \mathrm{Al}$. The reaction ${ }^{27} \mathrm{Al}\left({ }^{3} \mathrm{He}, \mathrm{x}\right){ }^{22} \mathrm{Na}$, proceeds through the direct reaction of the ${ }^{3} \mathrm{He}$ projectile with the core cluster ${ }^{25} \mathrm{Mg}$. On the other hand, the core cluster ${ }^{25} \mathrm{Mg}$ can be represented through the cluster model ${ }^{25} \mathrm{Mg}$ $\rightarrow{ }^{22} \mathrm{Na}+{ }^{3} \mathrm{H}$. Following the EMBNCM model the reaction ${ }^{27} \mathrm{Al}\left({ }^{3} \mathrm{He}, \mathrm{x}\right){ }^{22} \mathrm{Na}$ can be written as: ${ }^{3} \mathrm{He}+{ }^{27} \mathrm{Al} \rightarrow{ }^{3} \mathrm{He}+$ $\left({ }^{25} \mathrm{Mg}+\mathrm{d}\right) \rightarrow{ }^{3} \mathrm{He}+\left({ }^{22} \mathrm{Na}+\mathrm{t}\right)+\mathrm{d} \rightarrow{ }^{22} \mathrm{Na}+\mathrm{t}+{ }^{3} \mathrm{He}+\mathrm{d}$. In the exit channel of this reaction we have four fragments: ${ }^{22} \mathrm{Na}$, tritium, ${ }^{3} \mathrm{He}$, and deuteron.

Nuclear cluster structure of nuclei appears to be important to understand the reaction mechanisms. For high energies the projectile will interact with the core cluster of the target nucleus, and for low energies, the projectile will interact with the exterior cluster of the target nucleus. According to the EMBNCM model this is due to the fact that projectile needs more energy to interact, and to break-up the core cluster of the target nucleus, thus reaction $\mathrm{A}(\mathrm{a}, \mathrm{b}) \mathrm{B}$ involving projectile $\mathrm{a}$ and the core cluster of the nucleus A will occur at higher energies.

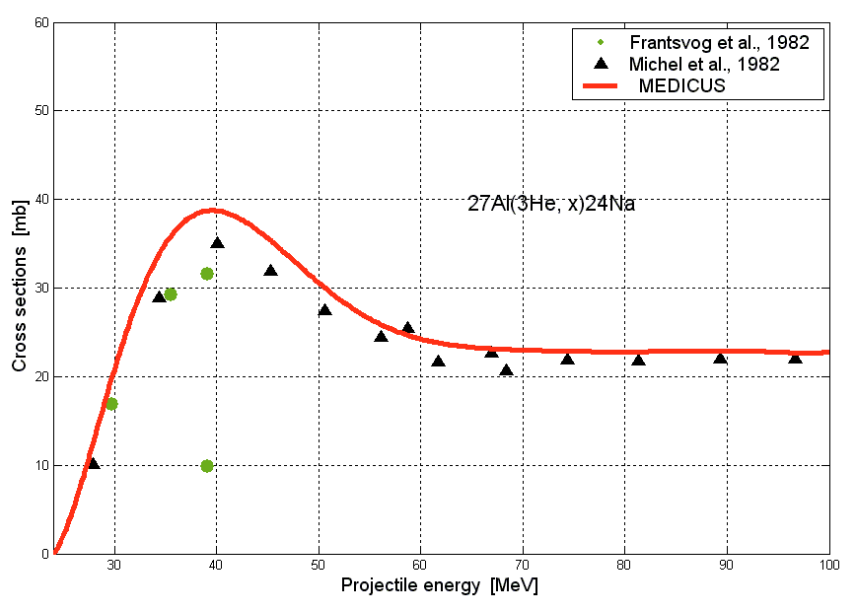

Fig. 3. Theoretical calculations of cross sections for the reaction ${ }^{27} \mathrm{Al}\left({ }^{3} \mathrm{He}, \mathrm{x}\right){ }^{24} \mathrm{Na}$ from MEDICUS code (line), and experimental data by Frantsvog et al. (1982) and Mighel et al. (1982) (symbols).

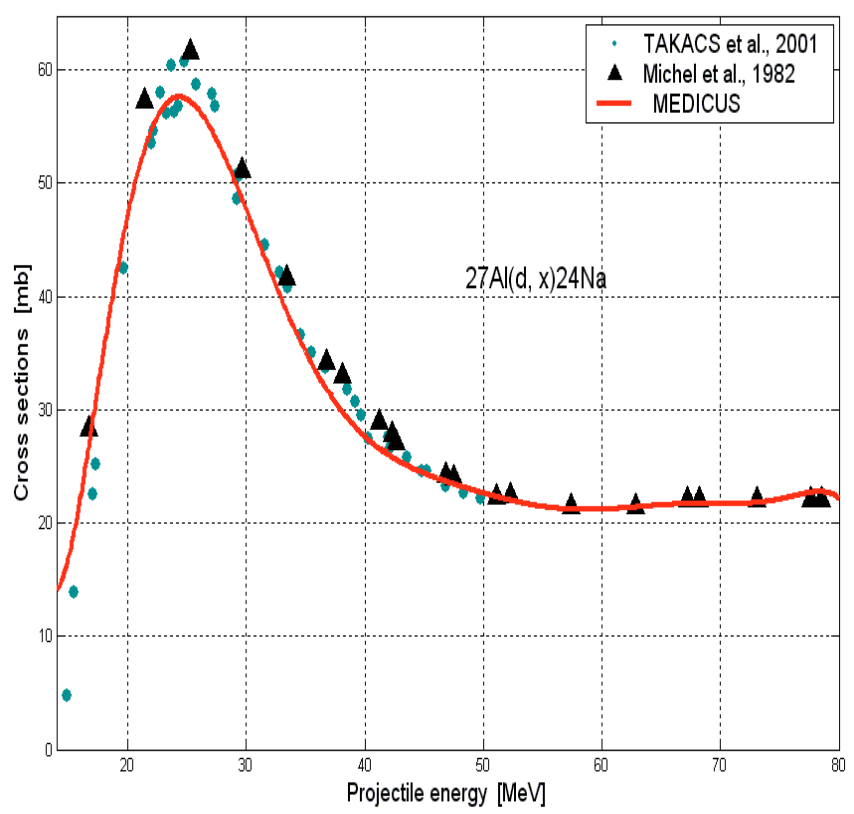

Fig. 4. Theoretical calculations of cross sections for the reaction ${ }^{27} \mathrm{Al}(\mathrm{d}, \mathrm{x})^{24} \mathrm{Na}$ from the MEDICUS code (line), and experimental data by Takacs et al. (2001) and Michel et al. (1982) (symbols).

For the low energies, projectile a will interact with exterior cluster of the target nucleus $\mathrm{A}$, and as a result the exterior cluster will be stripped from the target nucleus, and in the exit channel of this class of reactions we have the core cluster of the target nucleus, the exterior cluster of the nucleus, and the projectile. An example is the ${ }^{27} \mathrm{Al}\left({ }^{3} \mathrm{He}, \mathrm{x}\right){ }^{24} \mathrm{Na}$ reaction. Calculated cross sections for this reaction are presented in figure 3. As is predicted by the EMBNCM model, we can see that the reaction ${ }^{27} \mathrm{Al}\left({ }^{3} \mathrm{He}, \mathrm{x}\right){ }^{24} \mathrm{Na}$ occur at lower energies. The EMBNCM model can explain successfully direct reactions $(\mathrm{d}, \mathrm{x})$, involving as a target the ${ }^{27} \mathrm{Al}$ nucleus, and also other nuclei. In figure 4 are presented the calculated cross sections 
for the reaction ${ }^{27} \mathrm{Al}(\mathrm{d}, \mathrm{x}){ }^{24} \mathrm{Na}$. This reaction proceeds through the interaction of the deuteron with the exterior cluster of the ${ }^{27} \mathrm{Al}$ nucleus. The EMBNCM model predicts that on the exit channel of this reaction appears the core cluster of the target nucleus, projectile $\mathrm{d}$, and exterior cluster ${ }^{3} \mathrm{He}$ of the nucleus.

\section{Conclusions}

The code MEDICUS can be used successfully for calculating cross sections of nuclear reactions, and for nuclear cluster structure calculations. The EMBNCM model explains in nontraditional way reaction mechanisms. MEDICUS toolbox for MATLAB strength the MEDICUS power for calculations, combining and integrating it with MATLAB computing environment $[1,13]$. Reaction mechanisms are taken into account. Theoretical calculations of cross sections are in good agreement with experimental results.

I wish to thank the organizers of the ND2007 conference. I would like to thank Michael Whitburn and Pamela Dalby for proofreading the text of the summary.

\section{References}

1. A.I. Ibishi et al., MEDICUS toolbox for MATLAB, Proceedings of the First IEEE Benelux EMBS Symposium and Sixth Belgian Day on Biomedical Engineering, Brussels, Belgium, Dec. 7-8, 2006 (KVAB, Brussels), pp. 183-186.

2. A.I. Ibishi, MEDICUS code: application to nuclear medicine, Proceedings of the 22 $2^{\text {nd }}$ Annual BHPA Symposium "Physics in Medicine", Liège, Belgium, 2-3 February, 2007, edited by M.-T. Hoornaert, p. 78 .
3. A.I. Ibishi, A new method for calculation of nuclear cluster structure of nuclei, AIP Conf. Proc. 768 (Melville, New York, USA, 2005), pp. 395-397.

4. A.I. Ibishi, Proton $-{ }^{18} \mathrm{O}$ elastic scattering cross sections at high energies, AIP Conf. Proc. 768 (Melville, New York, USA, 2005), p. 408.

5. IUPAC, Compendium of Chemical terminology, 2nd edition (Blackwell Science, 1997).

6. A.I. Ibishi, A new method for calculation of nuclear cluster structure of nuclei, Proc. of the $19^{\text {th }}$ European conference on Few-Body Problems in Physics, Groningen, The Netherlands, August 23-27, 2004, p. 149.

7. A.I. Ibishi, Proton $-{ }^{18} \mathrm{O}$ elastic scattering cross sections at high energies, Proc. of the $19^{\text {th }}$ European Conference on Few-Body Problems in Physics, Groningen, The Netherlands, August 2327, 2004, p. 148.

8. C. Kalbach, Phys. Rev. C 33, 818 (1986).

9. T. Tamura et al., Phys. Rev. C 26, 379 (1982).

10. M. Blann, Phys. Rev. Lett. 27, 337 (1971).

11. N.A. Jelley, Fundamentals of nuclear physics (Cambridge University Press, Cambridge, New York, 1990).

12. D. Sutton, Radiology and Imaging for Medical Students, (Churchill Livingstone, $5^{\text {th }}$ edn., New York, 1988).

13. D. Redfern et al., The MATLAB 5 Handbook (Springer-Verlag, New York, 1998).

14. A.I. Ibishi et al., Recent development of the model code MEDICUS used for biomedical radioisotope production, Proceedings of the Fifth Belgian Day on Biomedical Engineering, Contactforum, Brussels, Belgium, 2006 (KVAB, Brussels), p. 56.

15. A.I. Ibishi et al., A new computer code MEDICUS for determination of cross sections of nuclear reactions used for biomedical radioisotope production, Proceedings of the $21^{\mathrm{st}}$ Annual Symposium of the BHPA 2006, Gent, Belgium, January 20-21 (2006), p. 121 . 